\title{
LA COMPLEJIDAD EN LAS ESTRUCTURAS DE LA VIOLENCIA: UNA MIRADA DESDE EL PROCESO EDUCATIVO
}

Darwin Reyes Solis

\section{Introducción}

El texto planteado para reflexión es un trabajo inacabado, es el inicio de un camino de investigación ${ }^{1}$. No se trata de un ensayo con resultados y conclusiones, sino del recuento de una hipótesis, una cartografía de lo que queremos investigar.

El tema de estudio es 'la violencia' y su interés es hacer las conexiones de ésta con la educación vivida en los sectores campesinos, periféricos de las urbes e indígenas de nuestro país. La motivación para realizar este documento surge de una larga trayectoria de trabajo con docentes y futuros docentes de los sectores nombrados, con quienes hemos debatido el tema de la violencia $y$, generalmente, terminamos referidos a nosotros mismos, a nuestros pasados, a nuestras experiencias educativas. El

* Docente de la Universidad Politécnica Salesiana. Licenciado en Filosofía y Master en Educación. 
recuerdo del pasado es un hecho recurrente para mostrar la violencia en la educación.

La pregunta que enmarca el trabajo de investigación es si la educación exige procesos violentos en sí misma. Violentar para poder incidir en quienes aprenden. ¿Es posible educar sin violencia? Esta interrogante nos interpela desde hace tiempo, en el trabajo con la educación popular, especialmente con la educación en los sectores rural e indígena. Y no porque éstos sean violentos, sino porque la realidad concreta en escuelas y colegios nos ha mostrado un camino de agresión que hemos recorrido en el proceso educativo.

Esa condición de agredidos, violentados, permanece en la memoria educativa y se reproduce como un sistema complejo que vive casi independiente de la voluntad de las personas. Es una violencia institucionalizada. Una violencia que tiene cuerpo propio y que se desplaza por las experiencias de los estudiantes y docentes de los sectores rurales.

\section{La violencia entendida como un método para enseñar}

Partimos de un hecho concreto. Vivimos en una sociedad marcada por sistemas violentos. Uno de los temas más amplios y complejos de tratar en sociedades contemporáneas es la seguridad de las personas, y ello porque los individuos tienen referencias de violencia que le intimidan, le producen miedo y angustia. No sólo se trata de la violencia en las calles, sino que tenemos violencia dentro de casa, dentro del aula y, muchas veces, dentro de nuestros propios cuerpos.

La violencia es una constante que la enfrentamos a lo largo de la vida. En este acápite analizaremos las experiencias de violencia y agresión vividas en el sistema 
de educación formal. Para nuestros planteamientos tomaremos una muestra de historias de vida de docentes de sectores campesinos e indígenas. Historias de vida que relatan sus experiencias violentas en la escuela y colegio.

La base de datos con la que hemos trabajado en estos años contiene más de 700 historias de vida, en las cuales se repiten los sistemas de violencia y agresión dentro de la educación formal y no formal.

Al parecer, hemos 'aprendido' que la educación es invasión violenta al mundo de la subjetividad del otro; ese presupuesto -que a mi entender es colonial- está muy marcado en los sistemas educativos, en los currículos ocultos, en las prácticas cotidianas de los centros educativos. Nos atrevemos a decir que es una memoria histórica compleja, 'cultural', un sistema de grabaciones colectivas difíciles de desmontar.

A continuación exponemos los testimonios de docentes campesinos, indígenas o de periferias urbanas recogidos en los años 2007-2010, en Cotopaxi, Chimborazo, Pichincha, Imbabura y Tungurahua, que cuentan sus historias de vida de cuando ellos/as eran educandos:

El primer grado, me hacia feo, estar enserada en el aula sin conocer a nadie ni siquiera a la señorita que tenia al frente mió en primer grado la señorita era mala nos gritaba si no entendíamos nos pegaba, o nos dejaba sin recreo diciendo que así tal vez aprenderán a ser obedientes e inteligentes.

También cuando nosotros los estudiantes no cumplíamos con los deberes y a los niños que eran mas molestos les tenía de rodillas frente al pizarrón hasta la hora de salir a recreo, si era después del recreo les tenía de rodillas hasta la hora de salir a la casa.

En segundo grado entre los niños del grado ya nos conocíamos y éramos amigos pero teníamos un profesor que 


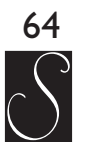

era bien estricto, no le gustaba el desorden, siempre a la hora de entrada nos revisaba el uniforme completo a el que no estaba bien uniformado le mandaba diciendo, volverán cuando tengas el uniforme completo, cuando no cumplíamos con los deberes no pegaba con la regla en la cabeza o en las manos.

En tercer grado teníamos una profesora que le gustaba gritar y no le gustaba repetir, cuando sorprendía a algún niño que no le estaba poniendo atención desde adelante le lanzaba el borrador a la cara y decía yo no soy payaso de nadie si no quiere estar en mi clase puede salir afuera. Esta profesora cuando nos hacía pasar al pizarrón a resolver los ejercicios de matemáticas y no podíamos nos cogía de los cabellos y nos daba contra el pizarrón, nos tenía parados hasta que termine de hacer el ejercicio y después de castigo nos mandaba a escribir cien veces tengo que poner atención a la clase.

En cuarto grado teníamos una profesora, ella era buena, le gustaba la puntualidad, que los niños seamos cumplidos con todos los deberes y obligaciones como estudiantes que estemos bien uniformados y que no llegemos atrasados cuando no hacíamos los deberes nos mandaba regresando a la casa y que al otro día vengamos con nuestro representante. En quinto grado estudiamos con la misma profesora.

En sexto grado teníamos una profesora que era irresponsable, la señorita llegaba de día y solamente le gustaba dictar ${ }^{2}$.

Historia de profesora (B.E.). Ayora, 2009

Las experiencias educativas que se han recogido tienen conexiones directas con sistemas violentos y métodos de enseñanza ligados al miedo para que los niños y jóvenes aprendan. En los testimonios recolectados entre los docentes, el tema de la violencia ha marcado a tal 
punto su vida, que los recuerdos de su educación, muy especialmente, de la primaria, hacen referencia a estructuras de dolor, agresión, gritos y golpes hacia los niños y niñas. No es casual que hagan referencia a sus profesores como personas 'estrictas' que violentaban permanentemente a los educandos. Eso sí, lo hacían para el bien de ellos (los educandos), porque les enseñaban cosas buenas.

Cuando yo ingresé al primer grado fue un maestro lojano y era el mas bueno de todos no tuve ni ningún problema.

Cuando yo pase a segundo grado me cambiaron de profesor y fue bien malo y egoísta porque nos maltrataba mucho Verbalmente cuando no hasiamos las tareas nos ortigaban nos llevaba a meter en el rio, si llegamos atrasados nos mandaba a la casa a traer al representante.

La parte de eso nos asia pegar con los padres nos dejaban sin el recreo parado en la esquina del aula hasta la hora de irse a la casa. Al siguiente dia nos hasian pasar al pizarrón ponían ejercicios muy difíciles. Cuando no podíamos nos tiraban con el borrador en la cara o en cualquier parte del cuerpo o también nos hasian gritar con todos los compañeros en voz bien alta y decía para que aprendamos y nos hacia bajar la moral.

Un dia el profesor vino bien enojado y nos puso una tarea bien difícil y era que casi todos no podíamos porque era difícil y nos dio 20 minutos para que terminemos, recogió todos los cuadernos y nos rompió todas las tareas que estaban mal y nos asian pasar por nombre que recoja el cuaderno a cada uno nos alaba del cabello y nos mandaba patiando contra el pizarrón y es asi que yo pude terminar mi primaria con muchos maltratos y desmoralización no fui solo sino que eramos entre diez compañeros que nos tenían vistitos en todo lo mas difícil. 
Este es mi triste historia que les puedo contar que fue muy triste y doloroza. Cuando me pongo analizar no se como pude soportar tantos maltratos.

Historia tomada de una profesora (I.G). Chimborazo, 2009

En los sistemas educativos rurales indígenas, se manejaban sistemas conductistas básicos, en donde el estímulo era la violencia y el resultado esperado era el aprendizaje. Es decir, se generaban procesos de violencia y miedo, para lograr aprendizajes o conocimientos específicos.

La relación entre aprendizaje y elementos de imposición psíquico o corporal es evidente. Al parecer es una herencia colonial. Esta afirmación es hipotética, pero encontramos elementos educativos en los que claramente podemos ver que los aprendizajes se logran al impartir 'respeto' a partir del miedo y aprendizajes que se graban gracias a factores de agresión corporal. Además, existe el elemento recurrente del 'insulto', la 'inferiorización', la 'anulación del sujeto', anhelando con esto dejar huella para motivar el aprendizaje y la necesidad de que los educandos entiendan que la educación es el camino de su 'salvación'; el único camino que les queda para ser sujetos sociales.

El recuerdo escolar siempre lleva a sufrimiento grabado en la memoria. Más que contenidos, al parecer, grabamos dolor.

Una materia que aparece como modelo de agresión es la matemática. Aunque en poblaciones indígenas también está presente el estudio del lenguaje castellano, pues eran kichwa hablantes.

Voy a contar, durante mi infancia educativa durante la escuela. A los 6 años de edad me he ingresado a la escuela de la parroquia en esa época no era necesario el requisito de haber cursado el jardín por lo tanto yo no sabía ni una sola letra. 
En mi primer año de escuela era muy tímido y no dominaba bién lo que es el idioma español yo solo hablaba kichwa y la profesora me maltrataba mal y yo no entendía lo que me enseñaba.

Terminó el primer año y yo me jale y no pasé de grado al 2do pero el siguiente año mis padres me matricularon de nuevo y yo ya sabía lo que me ivan a enseñar y salí bien hasta que estuve en tercer grado mi padre se falleció y quedamos huérfanos solo con mi madre. Y me cambié de escuela hacia la comunidad y culminé mi primaria.

En esos tres años de escuela en la comunidad era un buen estudiante porque pase dos años de escolta y el último año salí abanderado del pabellón de la bandera.

Historia de un profesor (T. F.). Cangahua, 2007

La maestra que tuvimos era super mala les pegaba a mis compañeros que vivían en el sector rural, les decía que apestaban a indios, los bañaba en las duchas de agua fría que había en la escuela, todos le teníamos un pavor inmenso, pero los indiecitos no entendían lo que decía.

Historia de una profesora (M.B.E.). Cayambe, 2007

Ahora bien, todo este sistema ha construido una memoria. ¿Será colectiva? Memoria que se reproduce en elementos cotidianos y vitales. No solamente se reproducen en sistemas educativos, sino en entornos familiares y sociales. Hasta el momento no logramos salir de esta manera de entender las relaciones con niños y jóvenes; no logramos abandonar la idea de que la educación es invasión violenta al mundo de la subjetividad del otro. 
Toda vivencia implica horizontes anteriores y posteriores, y se funde en última instancia con el continuum de las vivencias presentes, de antes y después, en la unidad de la corriente vivencial (Gadamer, 2000: 308).

Nos preguntamos cómo operan esos recuerdos; esas experiencias violentas vividas en su proceso educativo, experiencias que están marcadas en su memoria y producen dolor en sus vidas; ¿son fáciles de superar o procesar?, ¿será que las experiencias violentas repiten los sistemas educativos violentos? Recordemos que las repeticiones podemos hacerlas consciente o inconscientemente.

Además, cabe anotar que cuando los actuales profesores cuentan los maltratos y ejercicios agresivos realizados por sus maestros, los justifican diciendo que lo hacían 'para enseñar', 'porque eran estrictos', 'para que aprendan', 'por mi bien', 'me ha servido'. Es decir, aceptan esa realidad como algo que ayudó en el proceso educativo.

Los tres últimos años: recuerdo que hubo cambio de maestros los cuales eran muy estrictos y para mi parecer tenían un carácter muy fuerte.

El sistema de educación cambio ya no éramos tratados con amabilidad sino con una cultura de rigidez en las cosas que hacíamos, los deberes ya no eran cortos sino muy largos y con una letra muy clara y legible y quien no cumplía con estos procedimientos se exponía a ser pegado de maneras muy crueles como era el de que el compañero mas alto carge a quien no había echo los deberes y el profesor con una banda de elástica de la puerta de un carro procedía a pegarle quedando mis compañeros con mucho dolor y hasta en ciertas ocasiones por la fuerza del impacto se les reventaba la piel y con mucha crueldad se les ponía sal o mertiolate para que la herida deja- 
ra de sangrar mientras los demás compañeros estaban mirando con mucho miedo y asombro sobre lo que había pasado.

Sin duda estos tres últimos años fueron de mucho aprendizaje pero también de mucho dolor y miedo hacia a los maestros.

Historia de un profesor (J.T.). Cayambe, 2007

La violencia es un sistema muy complejo, pues participan profesores, padres, familia, iglesia, compañeros, 'patrones' y el azar de la vida. Al parecer son estructuras montadas desde la institucionalidad de un pueblo o cultura.

Y el cuarto, quinto, sexto año nos cambiaron con otros profesores y el profesor de dibujo era muy malo que asta no queríamos entrar en la clase de el, porque nos pegaba y nos jalaba del pelo por que no podíamos, también tuve una profesora de sociales que no era tan buena ni mala era regular y asi terminé y el año. No dentre al colegio porque mi madre no tuvo dinero, me pase en la casa tres años, a los catorce años me fui a trabajar en otavalo cuidando a una niña y mis patrones eran buenos asi que quise estudiar pero mis patrones no me dejaron porque ellos no tenián tiempo y no había quien se quede con la niña entonces me quede asta un año y luego me salí. [...] al quinto ahí pasó algo que no esperaba se enfermó mi madre a los quince días de estar enfermo se falleció mi madre, a mi me afectó muchísimo por ser la primera hija y luego ya no pensaba estudiar y ya no fui a la clase [...].

Historia de una profesora (G.Ch.). Cayambe, 2007

El primero, el segundo y el tercer año nunca puse empeño a estudiar, pero cuarto, quinto y sexto me gustaba estudiar nos cambiarón de profesor y terminamos con 
una profesora muy diferente para mí era muy buena, pero en estos años tenía mas trabajo porque tenía que llegar a tiempo a mi casa mi mamá me pegaba cuando no llegaba breve.

Cuando yo llegaba a mi casa me mandaba aser algún trabajo en la casa, luego me mandaba hacer los deberes casi nunca tenía tiempo para jugar con mi vecina siempre me tenía ocupada en alguna cosa o estudiando, era lo mejor para mi decía mi mamá.

Así termine la escuela, luego por bajos de recursos económicos no pude ir al colegio [...].

Historia de una profesora (M.F.). Cayambe, 2007

Podemos terminar este acápite con la hipótesis de que dichos sistemas educativos violentos se reproducen, cambian, pero, mantienen patrones de reproducción social y escolar. La violencia está con nosotros y debemos estudiarla, trabajarla. No solamente sentir pena o lástima de los violentados, sino que hemos de asumir una posición teórica, política y social para 'manejar' esta realidad que siempre está con la humanidad, pero que en nuestros sectores campesino-indígenas ha marcado una forma de entender los sistemas educativos y disciplinares de los niños y jóvenes.

\section{La violencia como estructura social compleja}

Pasemos ahora a un análisis más conceptual de la violencia, con la intención de acercarnos a su realidad, a su existir junto a nosotros.

La violencia como fenómeno humano presenta un mundo interno desde sistemas complejos e interrelacionados con otros fenómenos difíciles de develar. La ten- 
tación primeriza nos lleva a pensar que para iniciar una descomplejización de dichos fenómenos debemos definir el término para así tenerlo claro y trabajar desde allí. Pero, esa dimensión sólo revela ingenuidad al pensar que definiendo el término abarcamos la realidad, como si la definición fuese una ontología del ser. Esa es una noción bastante superada en nuestros tiempos. Definir no resuelve nada, sólo consuela al inocente. Definir sólo tranquiliza la saciedad de una mente ingenua. No vamos a definir nada, sólo vamos a enunciar ciertos elementos del debate, ciertos caminos tortuosos para repensar la violencia desde otros horizontes.

Superada la primera tentación vienen otras más finas, más elegantes, más 'modernas': 1 . Pensar unos fundamentos y explicar que la violencia es un objeto de estudio y definir ese objeto de estudio; 2. La idea de querer encontrar la verdad sobre la violencia, lo que ella 'es' como si ese 'es' fuese una verdad esencial palpable que se puede encontrar; 3. La vieja tendencia a solucionar el problema, antes de siquiera explicarlo o mostrarlo en sus dimensiones complejas, esa forma de querer llegar a soluciones rápidas, esa idea de que investigamos para solucionar problemas y, por lo tanto, debemos solucionar la violencia o la investigación misma no tendría sentido.

Al parecer no existe tal verdad en este mundo de complejidades e interrelaciones llamado violencia, no hay un objeto de estudio, sino que existen elementos inciertos, fronteras inestables, derivas vitales, conceptos inacabados. Esa es la complejidad de un mundo, dentro de otros mundos. Lo cual hace de la violencia un no lugar.

Para nuestro trabajo vamos a partir de algo concreto: las estructuras de la 'violencia' son un hecho real, existe. Es tan real que nos asusta, nos intimida, nos incomoda. 
Pero como toda estructura compleja la violencia no es homogénea ni lineal, sino que tiene varios rostros. Podemos decir que hay violencias, formas de ser diferentes de un mismo fenómeno. La violencia es multiplicidad de formas. Realicemos un acercamiento a esas formas, dejando claro que no es posible abarcar esta clasificación, sino que se trata de un acercamiento a su complejidad, para vislumbrar caminos a seguir en su comprensión.

La violencia es tan humana que puede ser entendida tanto desde dimensiones biológicas como desde estructuras culturales. Tratemos al inicio de la 'violencia fundamental', como el hecho concreto de que la vida se presenta en una lucha para sí misma y que se manifiesta desde una dimensión de defensa, de querer sostenerse y eso exige 'enfrentarse' al mundo y reproducirse en el mundo. Se trata de una 'base biológica' que se manifiesta en defensa de la vida y que se expresa como rupturas y choques frente a la alteridad y frente a la naturaleza, para así poder 'sacar la cabeza' y existir como sujeto.

La violencia fundamental, según Bergeret Jean, tendría que ver no con la agresividad, y menos aún con un sadismo de cualquier tipo, sino con el instinto de supervivencia [...]. Es la expresión de la persona que vive en peligro existencial, en peligro de muerte. Esta violencia da la imagen de sujeto bruto; surge porque la persona singular, aún antes de pretender construirse, debe existir, proteger su ser físico, su cuerpo, 'salvar su pellejo' y preservar así su posibilidad de convertirse ulteriormente en actor de su propia existencia rechazando el aplastamiento o la negación que se perfila (Wieviorka, 2006: 83).

Un hermoso ejemplo de esta violencia fundamental es el nacimiento de un nuevo sujeto que rompe el ritmo normal para existir como individuo. La violencia fundamental presenta rostros múltiples, por un lado se 
muestra creadora, dadora de vida, constructora de nuevas formas de ser. Por otro, es destrucción y enfrentamiento, es conflicto frente al mundo.

Podemos ver que la 'violencia fundamental' es parte de la vida misma, es tan humana como cualquier otra característica de nuestra existencia. No es buena, no es mala, simplemente existe con nosotros, es nuestra en el sentido existencial.

La violencia fundamental tiene rostros diversos. Por ejemplo, tenemos su rostro creador: la violencia creadora. Aquella que permite construir mundos, liberarnos de sistemas opresores, recrear lo humano con dignidad o construir un nuevo sujeto individual independiente de sus progenitores.

Fanon recuperaba este tipo de violencia para el proceso de descolonización, cuando analizaba las colonias americanas, sus sistemas esclavistas y las luchas 'violentas' de estos pueblos por construir su dignidad con horizontes propios. La misma dignidad que dirige a jóvenes como actores de un nuevo orden que con violencia permite que el viejo orden se desestructure y se destroce.

Pero, no solamente tenemos la violencia fundamental, sino que, como todo lo humano, la base biológica coexiste con las construcciones culturales e históricas. Pasemos entonces, a la construcción social de la violencia, aquellas formas de hacer violencia desde sujetos sociales frente al otro o frente a sí mismos. No se trata ya de la violencia fundamental, sino de una construcción cultural en las relaciones que realizo conmigo mismo y con el otro. Esa alteridad entendida no solamente como el 'alter sujeto', sino también como el 'alter naturaleza'; la violencia se da con estas dos dimensiones de la alteridad.

Esta violencia social tiene conexiones con la fundamental, pero no es lo mismo. Puede darse por: 1 . 
Carencia de sentido; 2. Una sobreexplotación del sentido hasta llegar a la destrucción por un sueño en el 'más allá'; 3. Por la presencia de un no-sujeto que logra vivir la destrucción del otro por obediencia; 4. Por crueldad o sadismo que logran hacer de la violencia un fin en sí mismo. Esta clasificación es utilizada por Wieviorka Michel en su texto La violencia y el sujeto. Tratemos de explicar cada una de ellas:

La violencia por carencia de sentido hace referencia a aquellas situaciones humanas en las que la acción misma pierde sentido, no se le puede dar un horizonte y entonces aparece la violencia como una forma de enfrentar ese sin sentido, eso que nos desborda y que nos hace perder el estado de ubicación. Por ejemplo, cuando grupos enfrentan violentamente al orden por agotamiento de las relaciones sociales y porque ya no ven sentido en las relaciones establecidas para el diálogo social.

En cambio, la violencia por sobreexplotación de sentido, refiere a aquellas situaciones humanas en las que el sentido se vuelve una totalidad absoluta y el sujeto y su voluntad desaparecen frente a esa totalidad. Haciendo que el sujeto sea capaz de aniquilarse o aniquilar en función de un sentido absoluto religioso, cultural o político. Como ejemplo de esta situación podemos ver los radicales políticos en autoatentados.

En la tercera dimensión de la violencia tenemos por la presencia de un no-sujeto, digamos que es lo contrario al sujeto, una construcción individual que obedece y por ello genera la destrucción del otro.

En esta perspectiva de la obediencia y de la sumisión a la autoridad, el verdugo no es ni sujeto, ni mucho menos, está atrapado en una lógica de la hipersubjetivización. Está definido por la pasividad, la indiferencia a sus pro- 
pios gestos; se reduce a ser el ejecutante de consignas burocráticas. Con él estamos frente a un ser desubjetivizado, que constituye un eslabón en un dispositivo que hace pensar en un sistema sin actores por fuera del o de los líderes (ibíd.: 78).

En una cuarta dimensión la violencia es pura búsqueda de placer, una forma de disfrute en la destrucción del otro, de la víctima. Aquí la violencia toma la constante de crueldad o sadismo.

En este texto no queremos analizar las dimensiones que toman la violencia o sus diferentes rostros, sino solamente los enunciamos para mostrar su multi y pluri dimensionalidad.

Volvamos a lo que nos compete, la violencia en relación a los procesos educativos. En el siguiente acápite vamos a ver las relaciones del castigo educativo para formar la imagen del cuerpo, de lo educado, de lo sano.

\section{Cuerpo, educación y violencia}

Nos preguntamos ¿Dónde depositamos toda esa violencia social? ¿Podemos dejarla fuera de nuestros cuerpos? ¿Es posible evitar que interfiera en nuestra única materialidad que poseemos como sí mismos: el cuerpo?

Por un lado, la violencia no solamente la expresamos en nuestras relaciones con el 'alter', sino que también la ejercemos en la construcción de nosotros mismos, en nuestro proceso individual, como sujetos, como cuerpos. Hay una fuerte costumbre de violentarnos a nosotros mismos, de depositar en nosotros la violencia. Un atentado consciente o inconsciente a nosotros mismos: una forma de ejercer la violencia, pero no hacia afuera, sino hacia dentro. 
En muchas experiencias de vida recogidas tenemos el relato de manejos corporales maltratantes: atentamos a nuestro cuerpo por formas de vivir que hemos aprendido y repetimos: maltratarnos y victimizarnos corporalmente.

Por otra parte, en los sistemas educativos, se hace referencia a los cuidados del cuerpo entendidos desde el control. Se trata de un control moral del cuerpo basado en políticas del miedo: la idea es que las personas teman el cuerpo, su uso y placeres para evitar la 'mala educación', la 'perdición', el 'salvajismo' y su proliferación en el cuerpo social.

La paradoja que se construye es: la educación construye la subjetividad desde la violencia corporal y psíquica, a eso denominamos 'educado-sano'. En cambio, si no 'disciplinamos' perdemos el sujeto y se hace 'mal educado-enfermo'.

Pero el cuidado del cuerpo implica más que lo biológico, se ve afectado por influencias sociales y culturales. Existe una marcada influencia de aspectos socioculturales, discursivos, en lo que tomamos como 'educado-sano' y en consecuencia lo que consideramos como cuerpo. Giddens toma la postura de Foucault sobre las llamadas tecnologías sociales para dar cuenta de esos aspectos de contaminación social sobre los cuerpos, donde las tecnologías son modalidades de intervención regular sobre el funcionamiento de los cuerpos; lo social determina aquello que es el cuerpo por medio de las significaciones discursivas, a su vez, dota al cuerpo de sentidos y es desde allí la tópica corporal del manejo y cuidado de sí.

En consecuencia, si es el cuidado del cuerpo humano una cuestión ligada al discurso, pues se trata de una decisión sobre el cuerpo la que tomamos en cada acto sobre él. Lo 'educado-sano' es cuestión de decidir sobre 
nuestro cuerpo, es la decisión sobre las acciones, sobre la alimentación, la ansiedad, el ejercicio (físico y mental) en busca de un bienestar. El bien-estar se referirá a una concepción moral del juego del bien y el mal y, por lo tanto, de una decisión ética sobre nuestra vida como posibilidad de expansión -bienestar- en contra de la muerte como posibilidad de extinción -malestar-.

Si es el cuerpo donde se enfocan nuestras posibilidades de 'educado-sano' es hacia esa tópica donde nos dirigimos con nuestra propuesta, no de un control del cuerpo por el miedo, sino, como una posición de optardecidir por el cuidado del cuerpo como un acto educativo valedero y humanizante.

\section{La necesidad de trabajar las relaciones entre educación y violencia}

Luego de un largo recorrido colaborando y compartiendo en educación popular, rural, e intercultural nos queda la sensación de una necesidad profunda de explicar, comprender y trabajar el problema de la violencia.

Las experiencias en nuestro recorrido educativo marcan una realidad de exagerado contenido violento. Al parecer, en algún momento, la violencia se insertó en el proceso educativo: el grito, la amenaza, la agresión física y psicológica, la prepotencia, se constituyeron en elementos de enseñanza aprendizaje. Nuestra hipótesis es que el origen de este fenómeno es eminentemente humano, pero se tornó en sistema educativo en la colonia. Desde allí se irradia una forma de hacer educación, una manera de generar aprendizajes desde la sumisión del cuerpo, desde la generación del miedo y desde la memoria de sufrimiento y dolor que hasta el momento se repiten y se recrean de diversas maneras. 
De las múltiples experiencias, además, podemos ver que existe una estrecha relación (también esto lo planteamos como hipótesis de trabajo) entre símbolos religiosos y formas de aprender. La idea de 'sufrir', de 'dolor' para ser 'buenos'; y, si la educación es para ser 'buenos', entonces se genera un lazo interior en la memoria colectiva entre lo bueno y el sufrir. Además, está presente este concepto recurrente en los sectores campesinos indígenas de que 'nada se puede conseguir sin sufrir', que 'todo es difícil en la vida'; por lo tanto, esfuerzo y sufrimiento son lo mismo y a ello si añadimos que la educación es -mucho más para estos sectores- un gran esfuerzo... ya podemos vislumbrar interrelaciones ideológicas y conceptuales. Dentro de esta misma hipótesis hemos encontrado símbolos religiosos que nos llevan a preguntarnos el sentido educativo y su historicidad dentro de los imaginarios sociales de los sectores populares. Por ejemplo: La Dolorosa del Colegio, madre de los estudiantes. Se trata de la Virgen llorando, con siete cuchillos en el corazón, los clavos con los que mataron a su hijo en la mano. Ella es la madre de los estudiantes.

Independiente de los símbolos religiosos tenemos una realidad que se repite: las relaciones entre sistemas de aprendizaje y metodologías violentas para lograrlo. Hasta el momento la respuesta ha sido prohibir toda violencia, considerarla mala y fea. Todo discurso educativo niega la violencia en sus construcciones conceptuales, pero las reproduce en sus prácticas. La violencia produce horror en las escuelas, pero sirve como sistema de aprendizaje. $\mathrm{Al}$ parecer se trata de una contradicción, pero no, solamente es una duplicación de lo real en un elemento lingüístico.

Nuestra última hipótesis es que debemos aceptar que la violencia es parte nuestra y desde allí debemos trabajar para 'manejarla'. La negación lo único que hace es fortalecerla, darle vida en el mundo de lo oscuro, de lo 
negado, de lo que está en el patio trasero. Pero ello no hace que desaparezca o que deje de actuar. Nuestro sistema educativo ha negado la violencia y así le ha dado más fuerza para actuar, porque nadie la puede pronunciar, porque vive independiente de lo que debemos hacer.

La violencia debe ser trabajada en la escuela como una dimensión humana de múltiples facetas. Consideramos que es muy similar al asunto de la sexualidad: al negarla, no la eliminamos, sino que la enviamos al lado oscuro, y desde allí los problemas son mayores. Vivimos una Era Victoriana en las instituciones educativas en el tema de la violencia.

Así llegamos a una paradoja insalvable: hemos dicho que la violencia es humana y que tienen infinidad de rostros. Hemos dicho también que el sistema colonial generó violencia para formar sujetos basándose en la agresión y así generó un sistema de repetición hasta nuestra época. Lo más sano -desde nuestra manera de comprender- es asumir la violencia y generar procesos educativos para vivirla de maneras dignas, sabiendo que su rostro destructivo estará siempre presente.

La propuesta es un nuevo lenguaje sobre la violencia. La actitud que especifica la dimensión de lo humano es el lenguaje; el lenguaje es diálogo, el lenguaje se convierte en médium de la realidad humana, es la imagen que construye el sentido de la realidad que el humano posee. Ese lenguaje mediador es el que debe liderar el 'enfrentar' la violencia como una realidad nuestra y darle nuevos horizontes de sentido. 


\section{Notas}

1 Reyes, Darwin: Violencia en la educación de sectores campesinos e indígenas andinos; Fundación Kawsay, no publicado.

2 Los testimonios de los profesores y aprendices fueron tomados de manera estricta. En este artículo hemos transcrito respetando los documentos originales.

\section{Bibliografía}

GADAMER HANS, George

2000 Verdad y método. Barcelona: Ed. Cátedra.

VARGAS LLOSA, Mario

1990 La verdad de las mentiras. Barcelona: Seix Barral.

WIEVIORKA, Michel

2006 “La violencia y el sujeto”. En: Assoun Paul Laurent, Zafiropoulos; Lógicas del síntoma Lógica pluridisciplinaria. Buenos Aires: Nueva Visión. 